\title{
Fetal Position above Umbilicus
}

National Cancer Institute

\section{Source}

National Cancer Institute. Fetal Position above Umbilicus. NCI Thesaurus. Code C92927.

A reference point for the fetal position when the fetal heart rate is detected above the mother's umbilicus. 